\title{
Measurement of Branching Fractions and Charge Asymmetries for Exclusive $B$ Decays to Charmonium
}

B. Aubert, ${ }^{1}$ R. Barate, ${ }^{1}$ D. Boutigny, ${ }^{1}$ F. Couderc,${ }^{1}$ Y. Karyotakis,${ }^{1}$ J. P. Lees,${ }^{1}$ V. Poireau, ${ }^{1}$ V. Tisserand, ${ }^{1}$ A. Zghiche,${ }^{1}$ E. Grauges-Pous, ${ }^{2}$ A. Palano, ${ }^{3}$ A. Pompili, ${ }^{3}$ J. C. Chen, ${ }^{4}$ N. D. Qi ${ }^{4}$ G. Rong,${ }^{4}$ P. Wang, ${ }^{4}$ Y. S. Zhu, ${ }^{4}$ G. Eigen, ${ }^{5}$ I. Ofte, ${ }^{5}$ B. Stugu, ${ }^{5}$ G. S. Abrams, ${ }^{6}$ A. W. Borgland, ${ }^{6}$ A. B. Breon, ${ }^{6}$ D. N. Brown, ${ }^{6}$ J. Button-Shafer, ${ }^{6}$ R. N. Cahn, ${ }^{6}$ E. Charles, ${ }^{6}$ C. T. Day, ${ }^{6}$ M. S. Gill, ${ }^{6}$ A. V. Gritsan, ${ }^{6}$ Y. Groysman, ${ }^{6}$ R. G. Jacobsen, ${ }^{6}$ R. W. Kadel, ${ }^{6}$ J. Kadyk, ${ }^{6}$ L. T. Kerth, ${ }^{6}$ Yu. G. Kolomensky, ${ }^{6}$ G. Kukartsev ${ }^{6}{ }^{\text {G. }}$ Lynch, ${ }^{6}$ L. M. Mir, ${ }^{6}$ P. J. Oddone, ${ }^{6}$ T. J. Orimoto, ${ }^{6}$ M. Pripstein, ${ }^{6}$ N. A. Roe, ${ }^{6}$ M. T. Ronan, ${ }^{6}$ W. A. Wenzel, ${ }^{6}$ M. Barrett, ${ }^{7}$ K. E. Ford, ${ }^{7}$ T. J. Harrison, ${ }^{7}$ A. J. Hart, ${ }^{7}$ C. M. Hawkes, ${ }^{7}$ S. E. Morgan, ${ }^{7}$ A. T. Watson, ${ }^{7}$ M. Fritsch, ${ }^{8}$ K. Goetzen, ${ }^{8}$ T. Held,${ }^{8}$ H. Koch,${ }^{8}$ B. Lewandowski,${ }^{8}$ M. Pelizaeus, ${ }^{8}$ T. Schroeder,${ }^{8}$ M. Steinke, J. T. Boyd,${ }^{9}$ N. Chevalier, ${ }^{9}$ W. N. Cottingham, ${ }^{9}$ M. P. Kelly, ${ }^{9}$ T. E. Latham, ${ }^{9}$ F. F. Wilson, ${ }^{9}$ T. Cuhadar-Donszelmann, ${ }^{10}$ C. Hearty,${ }^{10}$ N. S. Knecht, ${ }^{10}$ T. S. Mattison, ${ }^{10}$ J. A. McKenna,${ }^{10}$ D. Thiessen, ${ }^{10}$ A. Khan,${ }^{11}$ P. Kyberd, ${ }^{11}$ L. Teodorescu, ${ }^{11}$ A. E. Blinov, ${ }^{12}$ V. E. Blinov, ${ }^{12}$ V. P. Druzhinin, ${ }^{12}$ V. B. Golubev, ${ }^{12}$ V. N. Ivanchenko, ${ }^{12}$ E. A. Kravchenko, ${ }^{12}$ A. P. Onuchin, ${ }^{12}$ S. I. Serednyakov, ${ }^{12}$ Yu. I. Skovpen, ${ }^{12}$ E. P. Solodov, ${ }^{12}$ A. N. Yushkov, ${ }^{12}$ D. Best, ${ }^{13}$ M. Bruinsma,${ }^{13}$ M. Chao, ${ }^{13}$ I. Eschrich ${ }^{13}$ D. Kirkby, ${ }^{13}$ A. J. Lankford, ${ }^{13}$ M. Mandelkern, ${ }^{13}$ R. K. Mommsen, ${ }^{13}$ W. Roethel, ${ }^{13}$ D. P. Stoker, ${ }^{13}$ C. Buchanan, ${ }^{14}$ B. L. Hartfiel, ${ }^{14}$ A. J. R. Weinstein, ${ }^{14}$ S. D. Foulkes, ${ }^{15}$ J. W. Gary, ${ }^{15}$ O. Long, ${ }^{15}$ B. C. Shen, ${ }^{15}$ K. Wang, ${ }^{15}$ D. del Re,${ }^{16}$ H. K. Hadavand, ${ }^{16}$ E. J. Hill, ${ }^{16}$ D. B. MacFarlane, ${ }^{16}$ H. P. Paar, ${ }^{16}$ Sh. Rahatlou, ${ }^{16}$ V. Sharma, ${ }^{16}$ J. W. Berryhill, ${ }^{17}$ C. Campagnari, ${ }^{17}$ A. Cunha, ${ }^{17}$ B. Dahmes,${ }^{17}$ T. M. Hong, ${ }^{17}$ A. Lu, ${ }^{17}$ M. A. Mazur, ${ }^{17}$ J. D. Richman,,${ }^{17}$ W. Verkerke, ${ }^{17}$ T. W. Beck, ${ }^{18}$ A. M. Eisner, ${ }^{18}$ C. A. Heusch, ${ }^{18}$ J. Kroseberg, ${ }^{18}$ W. S. Lockman, ${ }^{18}$ G. Nesom, ${ }^{18}$ T. Schalk, ${ }^{18}$ B. A. Schumm, ${ }^{18}$

A. Seiden, ${ }^{18}$ P. Spradlin, ${ }^{18}$ D. C. Williams, ${ }^{18}$ M. G. Wilson, ${ }^{18}$ J. Albert,,${ }^{19}$ E. Chen, ${ }^{19}$ G. P. Dubois-Felsmann, ${ }^{19}$

A. Dvoretskii, ${ }^{19}$ D. G. Hitlin, ${ }^{19}$ I. Narsky ${ }^{19}$ T. Piatenko, ${ }^{19}$ F. C. Porter, ${ }^{19}$ A. Ryd,${ }^{19}$ A. Samuel, ${ }^{19}$ S. Yang,${ }^{19}$ S. Jayatilleke, ${ }^{20}$ G. Mancinelli, ${ }^{20}$ B. T. Meadows, ${ }^{20}$ M. D. Sokoloff, ${ }^{20}$ F. Blanc, ${ }^{21}$ P. Bloom, ${ }^{21}$ S. Chen, ${ }^{21}$ W. T. Ford,${ }^{21}$ U. Nauenberg, ${ }^{21}$

A. Olivas, ${ }^{21}$ P. Rankin, ${ }^{21}$ W. O. Ruddick,${ }^{21}$ J. G. Smith ${ }^{21}$ K. A. Ulmer, ${ }^{21}$ J. Zhang, ${ }^{21}$ L. Zhang, ${ }^{21}$ A. Chen, ${ }^{22}$ E. A. Eckhart, ${ }^{22}$ J. L. Harton, ${ }^{22}$ A. Soffer, ${ }^{22}$ W. H. Toki, ${ }^{22}$ R. J. Wilson, ${ }^{22}$ Q. Zeng, ${ }^{22}$ B. Spaan, ${ }^{23}$ D. Altenburg, ${ }^{24}$ T. Brandt, ${ }^{24}$ J. Brose, ${ }^{24}$ M. Dickopp, ${ }^{24}$ E. Feltresi,${ }^{24}$ A. Hauke,${ }^{24}$ H. M. Lacker, ${ }^{24}$ R. Nogowski, ${ }^{24}$ S. Otto ${ }^{24}$ A. Petzold,${ }^{24}$ J. Schubert, ${ }^{24}$ K. R. Schubert, ${ }^{24}$ R. Schwierz, ${ }^{24}$ J. E. Sundermann, ${ }^{24}$ D. Bernard, ${ }^{25}$ G. R. Bonneaud,${ }^{25}$ P. Grenier, ${ }^{25}$ S. Schrenk, ${ }^{25}$ Ch. Thiebaux, ${ }^{25}$ G. Vasileiadis, ${ }^{25}$ M. Verderi, ${ }^{25}$ D. J. Bard,${ }^{26}$ P. J. Clark,${ }^{26}$ F. Muheim, ${ }^{26}$ S. Playfer, ${ }^{26}$ Y. Xie, ${ }^{26}$ M. Andreotti, ${ }^{27}$ V. Azzolini, ${ }^{27}$ D. Bettoni, ${ }^{27}$ C. Bozzi,${ }^{27}$ R. Calabrese, ${ }^{27}$ G. Cibinetto, ${ }^{27}$ E. Luppi, ${ }^{27}$ M. Negrini, ${ }^{27}$ L. Piemontese, ${ }^{27}$ A. Sarti,${ }^{27}$ F. Anulli, ${ }^{28}$ R. Baldini-Ferroli, ${ }^{28}$ A. Calcaterra, ${ }^{28}$ R. de Sangro, ${ }^{28}$ G. Finocchiaro, ${ }^{28}$ P. Patteri, ${ }^{28}$ I. M. Peruzzi ${ }^{28}$ M. Piccolo, ${ }^{28}$ A. Zallo, ${ }^{28}$ A. Buzzo, ${ }^{29}$ R. Capra ${ }^{29}$ R. Contri, ${ }^{29}$ G. Crosetti, ${ }^{29}$ M. Lo Vetere, ${ }^{29}$ M. Macri, ${ }^{29}$ M. R. Monge, ${ }^{29}$ S. Passaggio, ${ }^{29}$ C. Patrignani, ${ }^{29}$ E. Robutti, ${ }^{29}$ A. Santroni, ${ }^{29}$ S. Tosi, ${ }^{29}$ S. Bailey, ${ }^{30}$ G. Brandenburg, ${ }^{30}$ K. S. Chaisanguanthum, ${ }^{30}$ M. Morii,${ }^{30}$ E. Won,${ }^{30}$ R. S. Dubitzky, ${ }^{31}$ U. Langenegger, ${ }^{31}$ J. Marks,${ }^{31}$ U. Uwer, ${ }^{31}$ W. Bhimji, ${ }^{32}$ D. A. Bowerman, ${ }^{32}$ P. D. Dauncey, ${ }^{32}$ U. Egede, ${ }^{32}$ J. R. Gaillard, ${ }^{32}$ G. W. Morton, ${ }^{32}$ J. A. Nash,${ }^{32}$ M. B. Nikolich, ${ }^{32}$ G. P. Taylor, ${ }^{32}$ M. J. Charles, ${ }^{33}$ G. J. Grenier, ${ }^{33}$ U. Mallik, ${ }^{33}$ J. Cochran, ${ }^{34}$ H. B. Crawley, ${ }^{34}$ J. Lamsa, ${ }^{34}$ W. T. Meyer, ${ }^{34}$ S. Prell,${ }^{34}$ E. I. Rosenberg, ${ }^{34}$ A. E. Rubin,${ }^{34}$ J. Yi,${ }^{34}$ N. Arnaud,${ }^{35}$ M. Davier, ${ }^{35}$ X. Giroux,${ }^{35}$ G. Grosdidier, ${ }^{35}$ A. Höcker, ${ }^{35}$ F. Le Diberder, ${ }^{35}$ V. Lepeltier, ${ }^{35}$ A. M. Lutz, ${ }^{35}$ T. C. Petersen, ${ }^{35}$ S. Plaszczynski,${ }^{35}$ M. H. Schune,${ }^{35}$ G. Wormser, ${ }^{35}$

C. H. Cheng, ${ }^{36}$ D. J. Lange, ${ }^{36}$ M. C. Simani,,${ }^{36}$ D. M. Wright, ${ }^{36}$ A. J. Bevan, ${ }^{37}$ C. A. Chavez, ${ }^{37}$ J. P. Coleman, ${ }^{37}$ I. J. Forster, ${ }^{37}$ J. R. Fry, ${ }^{37}$ E. Gabathuler, ${ }^{37}$ R. Gamet, ${ }^{37}$ D. E. Hutchcroft, ${ }^{37}$ R. J. Parry, ${ }^{37}$ D. J. Payne, ${ }^{37}$ C. Touramanis, ${ }^{37}$ C. M. Cormack, ${ }^{38}$ F. Di Lodovico, ${ }^{38}$ C. L. Brown, ${ }^{39}$ G. Cowan,${ }^{39}$ R. L. Flack, ${ }^{39}$ H. U. Flaecher, ${ }^{39}$ M. G. Green, ${ }^{39}$ P. S. Jackson, ${ }^{39}$ T. R. McMahon, ${ }^{39}$ S. Ricciardi, ${ }^{39}$ F. Salvatore, ${ }^{39}$ M. A. Winter,${ }^{39}$ D. Brown,${ }^{40}$ C. L. Davis, ${ }^{40}$ J. Allison, ${ }^{41}$ N. R. Barlow, ${ }^{41}$ R. J. Barlow, ${ }^{41}$ M. C. Hodgkinson, ${ }^{41}$ G. D. Lafferty, ${ }^{41}$ J. C. Williams, ${ }^{41}$ C. Chen, ${ }^{42}$ A. Farbin, ${ }^{42}$

W. D. Hulsbergen, ${ }^{42}$ A. Jawahery, ${ }^{42}$ D. Kovalskyi, ${ }^{42}$ C. K. Lae, ${ }^{42}$ V. Lillard, ${ }^{42}$ D. A. Roberts, ${ }^{42}$ G. Blaylock, ${ }^{43}$ C. Dallapiccola, ${ }^{43}$ S. S. Hertzbach, ${ }^{43}$ R. Kofler, ${ }^{43}$ V. B. Koptchev, ${ }^{43}$ T. B. Moore ${ }^{43}$ S. Saremi, ${ }^{43}$ H. Staengle, ${ }^{43}$ S. Willocq,${ }^{43}$ R. Cowan, ${ }^{44}$ K. Koeneke, ${ }^{44}$ G. Sciolla, ${ }^{44}$ S. J. Sekula, ${ }^{44}$ F. Taylor, ${ }^{44}$ R. K. Yamamoto, ${ }^{44}$ P. M. Patel,,${ }^{45}$ S. H. Robertson, ${ }^{45}$ A. Lazzaro, ${ }^{46}$ V. Lombardo ${ }^{46}$ F. Palombo ${ }^{46}$ J. M. Bauer,${ }^{47}$ L. Cremaldi, ${ }^{47}$ V. Eschenburg, ${ }^{47}$ R. Godang, ${ }^{47}$ R. Kroeger,${ }^{47}$

J. Reidy, ${ }^{47}$ D. A. Sanders, ${ }^{47}$ D. J. Summers, ${ }^{47}$ H. W. Zhao, ${ }^{47}$ S. Brunet, ${ }^{48}$ D. Côté ${ }^{48}$ P. Taras,${ }^{48}$ H. Nicholson, ${ }^{49}$ N. Cavallo, ${ }^{50, *}$ F. Fabozzi,${ }^{50, *}$ C. Gatto,${ }^{50}$ L. Lista,${ }^{50}$ D. Monorchio,${ }^{50}$ P. Paolucci,${ }^{50}$ D. Piccolo,${ }^{50}$ C. Sciacca, ${ }^{50}$ M. Baak,${ }^{51}$ H. Bulten, ${ }^{51}$ G. Raven, ${ }^{51}$ H. L. Snoek, ${ }^{51}$ L. Wilden, ${ }^{51}$ C. P. Jessop, ${ }^{52}$ J. M. LoSecco, ${ }^{52}$ T. Allmendinger,${ }^{53}$ G. Benelli, ${ }^{53}$ K. K. Gan ${ }^{53}$ K. Honscheid,${ }^{53}$ D. Hufnagel, ${ }^{53}$ H. Kagan, ${ }^{53}$ R. Kass,${ }^{53}$ T. Pulliam, ${ }^{53}$ A. M. Rahimi, ${ }^{53}$ R. Ter-Antonyan, ${ }^{53}$ Q. K. Wong, ${ }^{53}$ J. Brau, ${ }^{54}$ R. Frey, ${ }^{54}$ O. Igonkina, ${ }^{54}$ M. Lu, ${ }^{54}$ C. T. Potter, ${ }^{54}$ N. B. Sinev, ${ }^{54}$ D. Strom, ${ }^{54}$ E. Torrence, ${ }^{54}$ 
F. Colecchia, ${ }^{55}$ A. Dorigo, ${ }^{55}$ F. Galeazzi, ${ }^{55}$ M. Margoni, ${ }^{55}$ M. Morandin, ${ }^{55}$ M. Posocco, ${ }^{55}$ M. Rotondo, ${ }^{55}$ F. Simonetto,${ }^{55}$ R. Stroili,${ }^{55}$ C. Voci, ${ }^{55}$ M. Benayoun, ${ }^{56} \mathrm{H}$. Briand,${ }^{56} \mathrm{~J}$. Chauveau,${ }^{56} \mathrm{P}$. David ${ }^{56}$ Ch. de la Vaissière, ${ }^{56}$ L. Del Buono, ${ }^{56}$ O. Hamon, ${ }^{56}$ M. J. J. John, ${ }^{56}$ Ph. Leruste, ${ }^{56}$ J. Malcles ${ }^{56}$ J. Ocariz,${ }^{56}$ L. Roos,${ }^{56}$ G. Therin,${ }^{56}$ P. K. Behera,${ }^{57}$ L. Gladney, ${ }^{57}$ Q. H. Guo ${ }^{57}$ J. Panetta, ${ }^{57}$ M. Biasini,${ }^{58}$ R. Covarelli, ${ }^{58}$ M. Pioppi,${ }^{58}$ C. Angelini,${ }^{59}$ G. Batignani,${ }^{59}$ S. Bettarini,${ }^{59}$ M. Bondioli, ${ }^{59}$ F. Bucci, ${ }^{59}$ G. Calderini, ${ }^{59}$ M. Carpinelli, ${ }^{59}$ F. Forti, ${ }^{59}$ M. A. Giorgi, ${ }^{59}$ A. Lusiani, ${ }^{59}$ G. Marchiori, ${ }^{59}$ M. Morganti, ${ }^{59}$ N. Neri, ${ }^{59}$ E. Paoloni, ${ }^{59}$ M. Rama, ${ }^{59}$ G. Rizzo, ${ }^{59}$ G. Simi,${ }^{59}$ J. Walsh,${ }^{59}$ M. Haire, ${ }^{60}$ D. Judd,${ }^{60}$ K. Paick, ${ }^{60}$ D. E. Wagoner, ${ }^{60}$ N. Danielson, ${ }^{61}$ P. Elmer, ${ }^{61}$ Y.P. Lau,${ }^{61}$ C. Lu,${ }^{61}$ V. Miftakov, ${ }^{61}$ J. Olsen, ${ }^{61}$ A. J. S. Smith, ${ }^{61}$ A. V. Telnov, ${ }^{61}$ F. Bellini, ${ }^{62}$ G. Cavoto,${ }^{61,62}$ A. D'Orazio, ${ }^{62}$ E. Di Marco, ${ }^{62}$ R. Faccini, ${ }^{62}$ F. Ferrarotto, ${ }^{62}$ F. Ferroni, ${ }^{62}$ M. Gaspero, ${ }^{62}$ L. Li Gioi, ${ }^{62}$ M. A. Mazzoni, ${ }^{62}$ S. Morganti, ${ }^{62}$ M. Pierini, ${ }^{62}$ G. Piredda, ${ }^{62}$ F. Polci, ${ }^{62}$ F. Safai Tehrani, ${ }^{62}$ C. Voena, ${ }^{62}$ S. Christ ${ }^{63}$ H. Schröder ${ }^{63}$ G. Wagner ${ }^{63}$ R. Waldi, ${ }^{63}$ T. Adye,${ }^{64}$ N. De Groot, ${ }^{64}$ B. Franek, ${ }^{64}$ G. P. Gopal ${ }^{64}$ E. O. Olaiya, ${ }^{64}$ R. Aleksan, ${ }^{65}$ S. Emery ${ }^{65}$ A. Gaidot ${ }^{65}$ S. F. Ganzhur ${ }^{65}$ P.-F. Giraud, ${ }^{65}$ G. Hamel de Monchenault ${ }^{65}$ W. Kozanecki, ${ }^{65}$ M. Legendre, ${ }^{65}$ G. W. London ${ }^{65}$ B. Mayer, ${ }^{65}$ G. Schottt,${ }^{65}$ G. Vasseur, ${ }^{65}$ Ch. Yèche,${ }^{65}$ M. Zito, ${ }^{65}$ M. V. Purohit, ${ }^{66}$ A. W. Weidemann, ${ }^{66}$ J. R. Wilson,${ }^{66}$ F. X. Yumiceva, ${ }^{66}$ T. Abe,${ }^{67}$ M. Allen,${ }^{67}$ D. Aston, ${ }^{67}$ R. Bartoldus,${ }^{67}$ N. Berger, ${ }^{67}$ A. M. Boyarski, ${ }^{67}$ O. L. Buchmueller,${ }^{67}$ R. Claus,${ }^{67}$ M. R. Convery, ${ }^{67}$ M. Cristinziani, ${ }^{67}$ G. De Nardo ${ }^{67}$ J. C. Dingfelder, ${ }^{67}$ D. Dong, ${ }^{67}$ J. Dorfan,${ }^{67}$ D. Dujmic, ${ }^{67}$ W. Dunwoodie, ${ }^{67}$ S. Fan, ${ }^{67}$ R. C. Field, ${ }^{67}$ T. Glanzman, ${ }^{67}$ S. J. Gowdy ${ }^{67}$ T. Hadig ${ }^{67}$ V. Halyo, ${ }^{67}$ C. Hast ${ }^{67}$ T. Hryn'ova, ${ }^{67}$ W. R. Innes,${ }^{67}$ M. H. Kelsey, ${ }^{67}$ P. Kim ${ }^{67}$ M. L. Kocian, ${ }^{67}$ D. W. G. S. Leith, ${ }^{67}$ J. Libby, ${ }^{67}$ S. Luitz, ${ }^{67}$ V. Luth, ${ }^{67}$ H. L. Lynch,${ }^{67}$ H. Marsiske, ${ }^{67}$ R. Messner, ${ }^{67}$ D. R. Muller, ${ }^{67}$ C. P. O' Grady, ${ }^{67}$ V. E. Ozcan, ${ }^{67}$ A. Perazzo,${ }^{67}$ M. Perl, ${ }^{67}$ B. N. Ratcliff,,${ }^{67}$ A. Roodman, ${ }^{67}$ A. A. Salnikov, ${ }^{67}$ R. H. Schindler, ${ }^{67}$ J. Schwiening, ${ }^{67}$ A. Snyder, ${ }^{67}$ A. Soha, ${ }^{67}$ J. Stelzer ${ }^{67}$ J. Strube, ${ }^{54,67}$ D. Su, ${ }^{67}$ M. K. Sullivan,${ }^{67}$ J. Thompson, ${ }^{67}$ J. Va'vra, ${ }^{67}$ S. R. Wagner, ${ }^{67}$ M. Weaver, ${ }^{67}$ W. J. Wisniewski, ${ }^{67}$ M. Wittgen, ${ }^{67}$ D. H. Wright, ${ }^{67}$ A. K. Yarritu, ${ }^{67}$ C. C. Young, ${ }^{67}$ P. R. Burchat, ${ }^{68}$ A. J. Edwards, ${ }^{68}$ S. A. Majewski, ${ }^{68}$ B. A. Petersen, ${ }^{68}$ C. Roat, ${ }^{68}$ M. Ahmed, ${ }^{69}$ S. Ahmed, ${ }^{69}$ M. S. Alam, ${ }^{69}$ J. A. Ernst, ${ }^{69}$ M. A. Saeed, ${ }^{69}$ M. Saleem, ${ }^{69}$ F. R. Wappler, ${ }^{69}$ W. Bugg, ${ }^{70}$ M. Krishnamurthy, ${ }^{70}$ S. M. Spanier, ${ }^{70}$ R. Eckmann, ${ }^{71}$ H. Kim, ${ }^{71}$ J. L. Ritchie, ${ }^{71}$ A. Satpathy, ${ }^{71}$ R. F. Schwitters,${ }^{71}$ J. M. Izen, ${ }^{72}$ I. Kitayama, ${ }^{72}$ X. C. Lou,${ }^{72}$ S. Ye ${ }^{72}$ F. Bianchi,${ }^{73}$ M. Bona ${ }^{73}$ F. Gallo, ${ }^{73}$ D. Gamba, ${ }^{73}$ L. Bosisio, ${ }^{74}$ C. Cartaro,${ }^{74}$ F. Cossutti,${ }^{74}$ G. Della Ricca, ${ }^{74}$ S. Dittongo,${ }^{74}$ S. Grancagnolo,${ }^{74}$ L. Lanceri, ${ }^{74}$ P. Poropat,${ }^{74} \dagger$ L. Vitale, ${ }^{74}$ G. Vuagnin, ${ }^{74}$ F. Martinez-Vidal, ${ }^{2,75}$ R. S. Panvini, ${ }^{76}$ Sw. Banerjee,${ }^{77}$ B. Bhuyan,${ }^{77}$ C. M. Brown, ${ }^{77}$ D. Fortin, ${ }^{77}$ P. D. Jackson, ${ }^{77}$ R. Kowalewski, ${ }^{77}$ J. M. Roney,${ }^{77}$ R. J. Sobie, ${ }^{77}$ J. J. Back, ${ }^{78}$ P. F. Harrison,${ }^{78}$ G. B. Mohanty,${ }^{78}$ H. R. Band, ${ }^{79}$ X. Chen, ${ }^{79}$ B. Cheng, ${ }^{79}$ S. Dasu, ${ }^{79}$ M. Datta, ${ }^{79}$ A. M. Eichenbaum,,${ }^{79}$ K. T. Flood, ${ }^{79}$ M. Graham,${ }^{79}$ J. J. Hollar, ${ }^{79}$ J. R. Johnson, ${ }^{79}$ P. E. Kutter, ${ }^{79}$ H. Li,${ }^{79}$ R. Liu, ${ }^{79}$ A. Mihalyi, ${ }^{79}$ Y. Pan, ${ }^{79}$ R. Prepost, ${ }^{79}$ P. Tan,${ }^{79}$ J. H. von Wimmersperg-Toeller, ${ }^{79}$ J. Wu, ${ }^{79}$ S. L. Wu, ${ }^{79}$ Z. Yu, ${ }^{79}$ M. G. Greene, ${ }^{80}$ and H. Neal ${ }^{80}$

(BABAR Collaboration)

\author{
${ }^{1}$ Laboratoire de Physique des Particules, F-74941 Annecy-le-Vieux, France \\ ${ }^{2}$ Universitad Autonoma de Barcelona, E-08193 Bellaterra, Barcelona, Spain \\ ${ }^{3}$ Dipartimento di Fisica and INFN, Università di Bari, I-70126 Bari, Italy \\ ${ }^{4}$ Institute of High Energy Physics, Beijing 100039, China \\ ${ }^{5}$ Institute of Physics, University of Bergen, N-5007 Bergen, Norway \\ ${ }^{6}$ Lawrence Berkeley National Laboratory and University of California, Berkeley, California 94720, USA \\ ${ }^{7}$ University of Birmingham, Birmingham B15 2TT, United Kingdom \\ ${ }^{8}$ Institut für Experimentalphysik 1, Ruhr Universität Bochum, D-44780 Bochum, Germany \\ ${ }^{9}$ University of Bristol, Bristol BS8 1TL, United Kingdom \\ ${ }^{10}$ University of British Columbia, Vancouver, British Columbia, Canada V6T 1Z1 \\ ${ }^{11}$ Brunel University, Uxbridge, Middlesex UB8 3PH, United Kingdom \\ ${ }^{12}$ Budker Institute of Nuclear Physics, Novosibirsk 630090, Russia \\ ${ }^{13}$ University of California-Irvine, Irvine, California 92697, USA \\ ${ }^{14}$ University of California-Los Angeles, Los Angeles, California 90024, USA \\ ${ }^{15}$ University of California-Riverside, Riverside, California 92521, USA \\ ${ }^{16}$ University of California-San Diego, La Jolla, California 92093, USA \\ ${ }^{17}$ University of California-Santa Barbara, Santa Barbara, California 93106, USA \\ ${ }^{18}$ Institute for Particle Physics, University of California-Santa Cruz, Santa Cruz, California 95064, USA \\ ${ }^{19}$ California Institute of Technology, Pasadena, California 91125, USA \\ ${ }^{20}$ University of Cincinnati, Cincinnati, Ohio 45221, USA \\ ${ }^{21}$ University of Colorado, Boulder, Colorado 80309, USA \\ ${ }^{22}$ Colorado State University, Fort Collins, Colorado 80523, USA
}




\author{
${ }^{23}$ Institut fur Physik, Universität Dortmund, D-44221 Dortmund, Germany \\ ${ }^{24}$ Institut für Kern- und Teilchenphysik, Technische Universität Dresden, D-01062 Dresden, Germany \\ ${ }^{25}$ Ecole Polytechnique, LLR, F-91128 Palaiseau, France \\ ${ }^{26}$ University of Edinburgh, Edinburgh EH9 3JZ, United Kingdom \\ ${ }^{27}$ Dipartimento di Fisica and INFN, Università di Ferrara, I-44100 Ferrara, Italy \\ ${ }^{28}$ Laboratori Nazionali di Frascati dell'INFN, I-00044 Frascati, Italy \\ ${ }^{29}$ Dipartimento di Fisica and INFN, Università di Genova, I-16146 Genova, Italy \\ ${ }^{30}$ Harvard University, Cambridge, Massachusetts 02138, USA \\ ${ }^{31}$ Physikalisches Institut, Universität Heidelberg, Philosophenweg 12, D-69120 Heidelberg, Germany \\ ${ }^{32}$ Imperial College London, London SW7 2AZ, United Kingdom \\ ${ }^{33}$ University of Iowa, Iowa City, Iowa 52242, USA \\ ${ }^{34}$ Iowa State University, Ames, Iowa 50011-3160, USA \\ ${ }^{35}$ Laboratoire de l'Accélérateur Linéaire, F-91898 Orsay, France \\ ${ }^{36}$ Lawrence Livermore National Laboratory, Livermore, California 94550, USA \\ ${ }^{37}$ University of Liverpool, Liverpool L69 72E, United Kingdom \\ ${ }^{38}$ Queen Mary, University of London, London E1 4NS, United Kingdom \\ ${ }^{39}$ Royal Holloway and Bedford New College, University of London, Egham, Surrey TW20 OEX, United Kingdom \\ ${ }^{40}$ University of Louisville, Louisville, Kentucky 40292, USA \\ ${ }^{41}$ University of Manchester, Manchester M13 9PL, United Kingdom \\ ${ }^{42}$ University of Maryland, College Park, Maryland 20742, USA \\ ${ }^{43}$ University of Massachusetts, Amherst, Massachusetts 01003, USA \\ ${ }^{44}$ Laboratory for Nuclear Science, Massachusetts Institute of Technology, Cambridge, Massachusetts 02139, USA \\ ${ }^{45}$ McGill University, Montréal, Quebec, Canada H3A $2 T 8$ \\ ${ }^{46}$ Dipartimento di Fisica and INFN, Università di Milano, I-20133 Milano, Italy \\ ${ }^{47}$ University of Mississippi, University, Mississippi 38677, USA \\ ${ }^{48}$ Laboratoire René J. A. Lévesque, Université de Montréal, Montréal, Quebec, Canada H3C 3J7 \\ ${ }^{49}$ Mount Holyoke College, South Hadley, Massachusetts 01075, USA \\ ${ }^{50}$ Dipartimento di Scienze Fisiche and INFN, Università di Napoli Federico II, I-80126, Napoli, Italy \\ ${ }^{51}$ NIKHEF, National Institute for Nuclear Physics and High Energy Physics, NL-1009 DB Amsterdam, The Netherlands \\ ${ }^{52}$ University of Notre Dame, Notre Dame, Indiana 46556, USA \\ ${ }^{53}$ The Ohio State University, Columbus, Ohio 43210, USA \\ ${ }^{54}$ University of Oregon, Eugene, Oregon 97403, USA \\ ${ }^{55}$ Dipartimento di Fisica and INFN, Università di Padova, I-35131 Padova, Italy \\ ${ }^{56}$ Laboratoire de Physique Nucléaire et de Hautes Energies, Universités Paris VI et VII, F-75252 Paris, France \\ ${ }^{57}$ University of Pennsylvania, Philadelphia, Pennsylvania 19104, USA \\ ${ }^{58}$ Dipartimento di Fisica and INFN, Università di Perugia, I-06100 Perugia, Italy \\ ${ }^{59}$ Dipartimento di Fisica, Scuola Normale Superiore and INFN, Università di Pisa, I-56127 Pisa, Italy \\ ${ }^{60}$ Prairie View A\&M University, Prairie View, Texas 77446, USA \\ ${ }^{61}$ Princeton University, Princeton, New Jersey 08544, USA \\ ${ }^{62}$ Dipartimento di Fisica and INFN, Università di Roma La Sapienza, I-00185 Roma, Italy \\ ${ }^{63}$ Universität Rostock, D-18051 Rostock, Germany \\ ${ }^{64}$ Rutherford Appleton Laboratory, Chilton, Didcot, Oxon OX11 OQX, United Kingdom \\ ${ }^{65}$ DSM/Dapnia, CEA/Saclay, F-91191 Gif-sur-Yvette, France \\ ${ }^{66}$ University of South Carolina, Columbia, South Carolina 29208, USA \\ ${ }^{67}$ Stanford Linear Accelerator Center, Stanford, California 94309, USA \\ ${ }^{68}$ Stanford University, Stanford, California 94305-4060, USA \\ ${ }^{69}$ State University of New York, Albany, New York 12222, USA \\ ${ }^{70}$ University of Tennessee, Knoxville, Tennessee 37996, USA \\ ${ }^{71}$ University of Texas-Austin, Austin, Texas 78712, USA \\ ${ }^{72}$ University of Texas-Dallas, Richardson, Texas 75083, USA \\ ${ }^{73}$ Dipartimento di Fisica Sperimentale and INFN, Università di Torino, I-10125 Torino, Italy \\ ${ }^{74}$ Dipartimento di Fisica and INFN, Università di Trieste, I-34127 Trieste, Italy \\ ${ }^{75}$ Universitad de Valencia, E-46100 Burjassot, Valencia, Spain \\ ${ }^{76}$ Vanderbilt University, Nashville, Tennessee 37235, USA \\ ${ }^{77}$ University of Victoria, Victoria, British Columbia, Canada V8W $3 P 6$ \\ ${ }^{78}$ Department of Physics, University of Warwick, Coventry CV4 7AL, United Kingdom \\ ${ }^{79}$ University of Wisconsin, Madison, Wisconsin 53706, USA \\ ${ }^{80}$ Yale University, New Haven, Connecticut 06511, USA \\ (Received 22 December 2004; published 14 April 2005)
}


We report measurements of branching fractions and charge asymmetries of exclusive decays of neutral and charged $B$ mesons into two-body final states containing a charmonium state and a light strange meson. The charmonium mesons considered are $J / \psi, \psi(2 S)$ and $\chi_{c 1}$, and the light meson is either $K$ or $K^{*}$. We use a sample of about $124 \times 10^{6} B \bar{B}$ pairs collected with the BABAR detector at the PEP-II storage ring at the Stanford Linear Accelerator Center.

DOI: 10.1103/PhysRevLett.94.141801

PACS numbers: 13.25.Hw, 11.30.Er, 12.15.Hh

Nonleptonic decays of $B$ mesons provide tests of both strong- and weak-interaction dynamics. Decays $B \rightarrow$ $(c \bar{c}) K^{(*)}$ are particularly illuminating as they involve three kinds of mesons: one with a heavy quark and a light quark, one with two heavy quarks, and one with two light quarks. A better description of the long distance nonpertubative aspects of QCD is indeed necessary to improve our understanding of heavy meson weak decays and more particularly our knowledge of the quark mixing matrix [1]. Phenomenological models (see Refs. [2-12] of Ref. [2]) give estimates for branching fractions and for ratios of the decays to $K$ and $K^{*}$. Some branching fractions and ratios have been reported (early measurements can be found in $[3,4]$ ) but others have not, so more stringent tests of the models are possible. The standard model predicts small differences between the branching fractions for positive and negative $B$ mesons, i.e., small direct $C P$ violation [5]. Large charge asymmetries would be evidence for new physics. Limits on direct $C P$ violation would constrain extensions of the standard model. Very few charge asymmetry measurements have been reported in $B \rightarrow(c \bar{c}) K^{(*)}$ modes [6]. The decay processes studied in this Letter are listed in Table I.

The data sample used in this analysis contains $124 \times$ $10^{6} B \bar{B}$ events collected with the $B A B A R$ detector at the PEP-II asymmetric $e^{+} e^{-}$storage ring. This represents a total integrated luminosity of $112.4 \mathrm{fb}^{-1}$ taken on the $Y(4 S)$ resonance. The BABAR detector is described in detail elsewhere [7]. Surrounding the interaction point, a five-layer double-sided silicon vertex tracker (SVT) provides precise reconstruction of track angles and $B$ decay vertices. A 40-layer drift chamber $(\mathrm{DCH})$ provides measurements of the transverse momenta of charged particles. An internally reflecting ring-imaging Cherenkov detector (DIRC) is used for particle identification. A CsI(Tl) crystal electromagnetic calorimeter (EMC) is used to detect photons and electrons. The calorimeter is surrounded by a 1.5-T magnetic field. The flux return is instrumented with resistive plate chambers (RPC) used for muon and neutral-hadron identification.

Multihadron events are selected by demanding a minimum of three reconstructed charged tracks in the polarangle range $0.41<\theta<2.54 \mathrm{rad}$, where $\theta$ is defined in the laboratory frame. Charged tracks must be reconstructed in the DCH and are required to originate within $1.5 \mathrm{~cm}$ of the beam in the plane transverse to it and within $10 \mathrm{~cm}$ of the beam spot along the beam direction. Events are required to have a primary vertex within $0.5 \mathrm{~cm}$ of the average position of the interaction point in the plane transverse to the beam line, and within $6 \mathrm{~cm}$ longitudinally. Charged tracks are required to include at least $12 \mathrm{DCH}$ hits and to have a transverse momentum $p_{T}>100 \mathrm{MeV} / c$. Photons are reconstructed from EMC clusters. The lateral energy profile (LAT) [8] is used to discriminate electromagnetic from hadronic clusters. Photons are required to have a minimum energy of $30 \mathrm{MeV}$, to satisfy LAT $<0.8$, and to be in the fiducial volume $0.41<\theta<2.41 \mathrm{rad}$. Electron candidates are selected using information from the EMC (LAT and Zernike moment $A_{42}$ [9]), the ratio of the energy measured in the EMC to the momentum measured by the tracking system, the energy loss in the drift chamber, and the Cherenkov angle measured in the DIRC. Electrons are also required to be in the fiducial volume $0.41<\theta<$ $2.41 \mathrm{rad}$. Muon candidates are selected using information from the EMC (energy deposition consistent with a minimum ionizing particle) and the distribution of hits in the RPC. Muons are required to be in the fiducial volume $0.3<\theta<2.7 \mathrm{rad}$. We select charged kaon and pion candidates using information from the energy loss in the SVT and DCH, and the Cherenkov angle measured in the DIRC. Kaon candidates are required to be in the fiducial volume $0.45<\theta<2.45 \mathrm{rad}$.

The selection has been optimized by maximizing the ratio $S / \sqrt{S+B}$, where $S$ and $B$ are the number of expected signal and background events obtained from Monte Carlo simulation. The $J / \psi$ candidates are required to have an invariant mass $2.95<M_{e^{+} e^{-}}<3.14 \mathrm{GeV} / c^{2}$ or $3.06<$ $M_{\mu^{+} \mu^{-}}<3.14 \mathrm{GeV} / c^{2}$ for $J / \psi \rightarrow e^{+} e^{-}$or $J / \psi \rightarrow$ $\mu^{+} \mu^{-}$decays, respectively. The $\psi(2 S)$ candidates are required to have an invariant mass $3.44<M_{e^{+} e^{-}}<$ $3.74 \mathrm{GeV} / c^{2}$ or $3.64<M_{\mu^{+} \mu^{-}}<3.74 \mathrm{GeV} / c^{2}$ for $\psi(2 S) \rightarrow e^{+} e^{-}$or $\psi(2 S) \rightarrow \mu^{+} \mu^{-}$decays, respectively.

TABLE I. Decay processes studied in this analysis. The $(c \bar{c})$ resonance is either a $J / \psi, \psi(2 S)$, or $\chi_{c 1}$. For all processes, when relevant, we use the secondary-decay modes $J / \psi, \psi(2 S) \rightarrow$ $\ell^{+} \ell^{-}, \chi_{c 1} \rightarrow J / \psi \gamma$, and $K_{S}^{0} \rightarrow \pi^{+} \pi^{-}, \pi^{0} \rightarrow \gamma \gamma$.

\begin{tabular}{lc}
\hline \hline Decay channel & Secondary $K^{*}$ decay mode \\
\hline$B^{0} \rightarrow(c \bar{c}) K^{* 0}$ & $K^{* 0} \rightarrow K^{+} \pi^{-}, K_{S}^{0} \pi^{0}$ \\
$B^{+} \rightarrow(c \bar{c}) K^{*+}$ & $K^{*+} \rightarrow K^{+} \pi^{0}, K_{S}^{0} \pi^{+}$ \\
$B^{0} \rightarrow(c \bar{c}) K_{S}^{0}$ & \\
$B^{+} \rightarrow(c \bar{c}) K^{+}$ & \\
\hline \hline
\end{tabular}


For $J / \psi, \psi(2 S) \rightarrow e^{+} e^{-}$decays, electron candidates are combined with photon candidates in order to recover some of the energy lost through bremsstrahlung. In the $\chi_{c 1}$ reconstruction, $J / \psi$ candidates are selected as described above. The associated $\gamma$ has to satisfy LAT $<0.8, A_{42}<$ 0.15 and has to have an energy greater than $0.15 \mathrm{GeV}$. The $\chi_{c 1}$ candidates are required to satisfy $0.35<M_{\ell^{+} \ell^{-} \gamma}-$ $M_{\ell^{+} \ell^{-}}<0.45 \mathrm{GeV} / c^{2}$, where $\ell$ represents an electron or a muon. The $\pi^{0} \rightarrow \gamma \gamma$ candidates are required to satisfy $0.113<M_{\gamma \gamma}<0.153 \mathrm{GeV} / c^{2}$. Both photons have to satisfy LAT $<0.8$. The energy of the soft photon has to be greater than $0.050 \mathrm{GeV}$ and the energy of the hard photon has to be greater than $0.150 \mathrm{GeV}$. The $K_{S}^{0} \rightarrow \pi^{+} \pi^{-}$candidates are required to satisfy $0.489<M_{\pi^{+} \pi^{-}}<$ $0.507 \mathrm{GeV} / c^{2}$. In addition, the $K_{S}^{0}$ flight distance defined as the distance between the reconstructed $B$ and $K_{S}^{0}$ vertices must exceed $1 \mathrm{~mm}$, and the angle between the $K_{S}^{0}$ momentum and its flight direction in the plane transverse to the beam axis must be less than $0.2 \mathrm{rad}$. The $K^{* 0}$ and $K^{*+}$ candidates are required to satisfy $0.796<M_{K \pi}<$ $0.996 \mathrm{GeV} / c^{2}$ and $0.792<M_{K \pi}<0.992 \mathrm{GeV} / c^{2}$, respectively. In addition, for the sake of suppressing background from events with soft pions, for channels having a $\pi^{0}$ in the final state, the cosine of the angle between the $K$ momentum and the $B$ momentum in the $K^{*}$ rest frame has to be less than 0.8 .

The $B$ candidates are reconstructed by combining charmonium and kaon candidates and are characterized by two kinematic variables: the difference between the reconstructed energy of the $B$ candidate and the beam energy in the center-of-mass frame $\Delta E=E_{B}^{*}-E_{\text {beam }}^{*}$, and the beam energy-substituted mass $m_{\mathrm{ES}}$, defined as $m_{\mathrm{ES}} \equiv$ $\sqrt{E_{\text {beam }}^{* 2}-\mathbf{p}_{B}^{* 2}}$, where the $*$ refers to quantities in the center of mass and $\mathbf{p}_{B}$ is the $B$ momentum. For a correctly reconstructed $B$ meson, $\Delta E$ is expected to peak at zero and the energy-substituted mass $m_{\mathrm{ES}}$ at the $B$ meson mass, $5.279 \mathrm{GeV} / c^{2}$. Only one reconstructed $B$ meson is allowed per event. For events that have multiple candidates, the candidate having the smallest $|\Delta E|$ is chosen. The analysis is performed in a region of the $m_{\mathrm{ES}}$ vs $\Delta E$ plane defined by $5.2<m_{\mathrm{ES}}<5.3 \mathrm{GeV} / c^{2}$ and $-0.12<\Delta E<0.12 \mathrm{GeV}$. A $\Delta E$ channel-dependent signal region is subsequently defined. The $m_{\mathrm{ES}}$ distributions within the $\Delta E$ signal region for candidate events are shown on Fig. 1.

There are two components to the residual background in the $\Delta E$ signal region: the combinatorial background and a peaking component (component of the background that peaks at the same values of $\Delta E$ and $m_{\mathrm{ES}}$ as the signal). The number of signal events $N_{S}$ is determined from the number of candidate events, $N_{\text {cand }}$, after subtracting the peaking background. For this purpose, the $m_{\mathrm{ES}}$ distribution within the $\Delta E$ signal region is fitted to the sum of an ARGUS function [4], which models the combinatorial background, and a Gaussian function. The value of $N_{\text {cand }}$
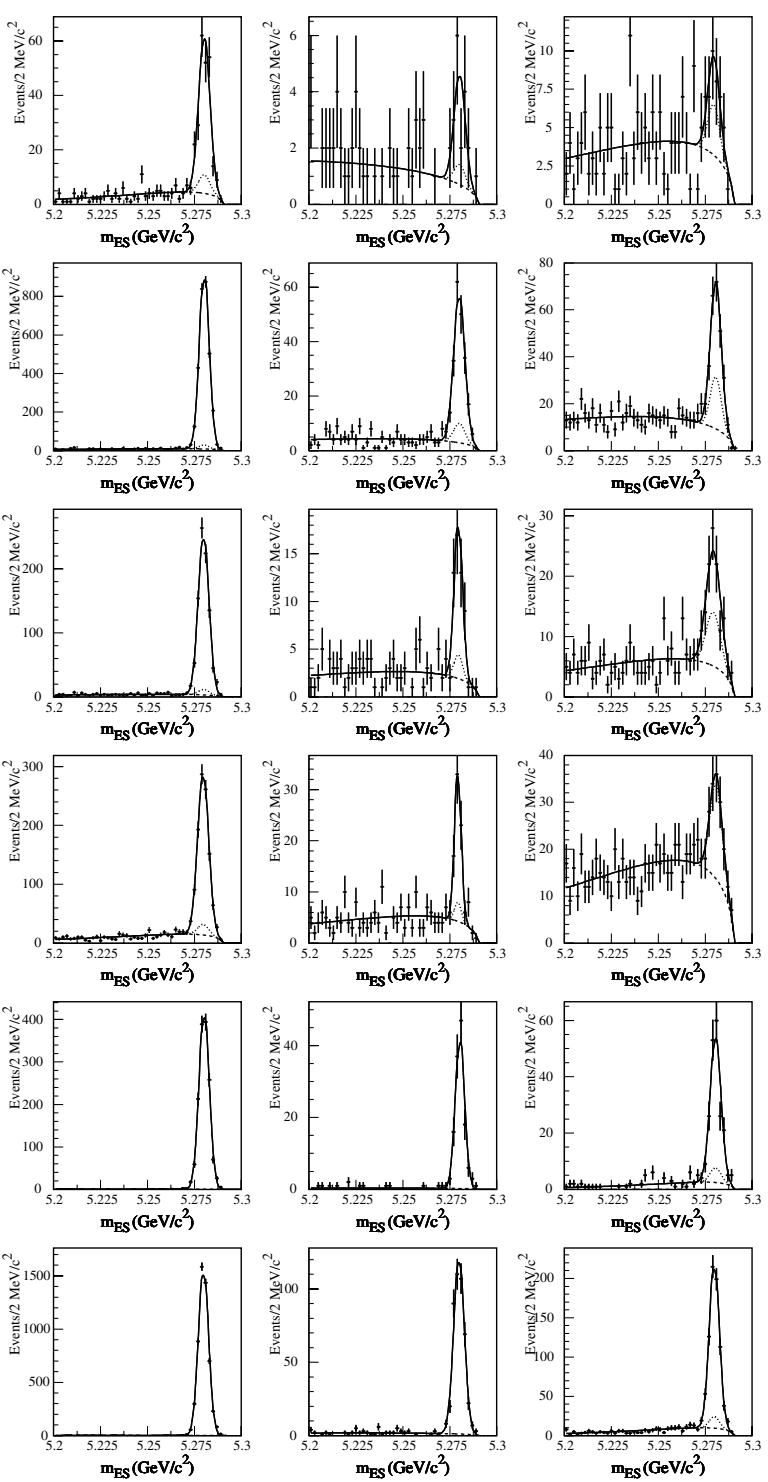

FIG. 1. $m_{\mathrm{ES}}$ distributions and fits within the $\Delta E$ signal region. From left to right, the columns show the distributions for the $J / \psi, \psi(2 S)$, and $\chi_{c 1}$ channels. From top to bottom, the rows show the distributions for the $B \rightarrow(c \bar{c}) K^{* 0}\left(K_{S}^{0} \pi^{0}\right), B \rightarrow(c \bar{c})$ $K^{* 0}\left(K^{+} \pi^{-}\right), B \rightarrow(c \bar{c}) K^{*+}\left(K_{S}^{0} \pi^{+}\right), B \rightarrow(c \bar{c}) K^{*+}\left(K^{+} \pi^{0}\right)$, $B \rightarrow(c \bar{c}) K_{S}^{0}$ and $B \rightarrow(c \bar{c}) K^{+}$decay modes. The dashed and dotted lines show the combinatorial and peaking backgrounds, respectively.

is given by the integral of the Gaussian component. There are two contributions to the peaking background. The first is the cross-feed component that is due to $B \rightarrow(c \bar{c}) K^{*}$ events from the three channels other than the one under consideration. The second contribution is from other $B$ decays with a $J / \psi$ or $\psi(2 S)$ in the final state. To determine the extent of peaking background, the $m_{\mathrm{ES}}$ distribution for simulated $B \bar{B}$ events is fitted within the $\Delta E$ signal region by an ARGUS function and a Gaussian function. The peaking background is taken as the integral of the Gaussian portion. 
The branching fractions are obtained as

$$
B F \equiv \frac{N_{S}}{N_{B \bar{B}} \times \epsilon \times f},
$$

where $N_{B \bar{B}}$ is the number of $B \bar{B}$ events, $\epsilon$ is the selection efficiency, and $f$ is the total secondary branching fraction. For channels with a $K^{*}$ in the final state, the cross-feed contribution depends on the branching fractions that are being measured. It was estimated by an iterative procedure and found to be small.

The systematic errors arise from the uncertainty on the number of $B \bar{B}$ events $(1.1 \%)$, the secondary branching fractions (taken from Ref. [10]), the estimate of the selection efficiency, and the knowledge of the background. For the tracking efficiency, an error of $1.3 \%$ per track has been used. For the particle identification efficiency, the systematic error varies between $0.2 \%$ and $3.7 \%$. The uncertainty on the detection and energy measurement of photons is $2.5 \%$, common to all channels, plus a small channeldependent correction. The uncertainty on the $\pi^{0}$ reconstruction is $5.0 \%$ for all channels, plus a channel-dependent correction. The overall selection efficiency depends on the angular distribution. The efficiency can be written as $\epsilon=$ $a+\left|A_{0}\right|^{2} b$, where $a$ and $b$ are obtained from the $K^{*}$ helicity angle by $a=3 / 4 \int\left(1-\cos ^{2} \theta_{K^{*}}\right) \epsilon\left(\theta_{K^{*}}\right) \times$ $\sin \left(\theta_{K^{*}}\right) d \theta_{K^{*}} \quad$ and $\quad b=3 / 4 \int\left(3 \cos ^{2} \theta_{K^{*}}-1\right) \epsilon\left(\theta_{K^{*}}\right) \times$ $\sin \left(\theta_{K^{*}}\right) d \theta_{K^{*}}$, and $\left|A_{0}\right|^{2}$ is the fraction of the longitudinal $K^{*}$ polarization [11]. The values of $a$ and $b$ are obtained from simulation. Using an error for $\left|A_{0}\right|^{2}$ varying between $7 \%$ and $15 \%$ [11] depending on the final state, we obtain a systematic uncertainty on the efficiency and consequently on the branching fractions varying from $3.4 \%$ to $8.6 \%$. In the default fit, the shape parameter of the ARGUS function is not constrained. To determine a systematic error due to the combinatorial background, a second fit with the shape parameter of the ARGUS function fixed to the value obtained from fitting the data in the $\Delta E$ sideband region was performed. The systematic uncertainty on the combinatorial background has been taken as $50 \%$ of the difference between the number of events obtained from the default fit and from the second fit. For the cross-feed component to the peaking background, the uncertainty of the corresponding branching fractions, taken from Ref. [10], has been assigned as the systematic error. For the contribution coming from other $B$ decays with a $J / \psi$ or a $\psi(2 S)$ in the final state, a 50\% error has been assigned, accounting for the poor knowledge of the branching fractions of the contributing decay modes. Overall, the dominant contribution to the systematic error is from the secondary-decay branching fractions in the case of $\psi(2 S) K^{(*)}$ and $\chi_{c 1} K$, the efficiency determination in the case of $J / \psi K^{(*)}$, and the background subtraction in the case of $\chi_{c 1} K^{*}$.

Assuming isospin invariance in the $B \rightarrow$ charmonium $K$ $\left(K^{*}\right)$ decays, we compute $R^{+/ 0}$, the ratio $\Gamma(Y(4 S) \rightarrow$ $\left.B^{+} B^{-}\right) / \Gamma\left(\Upsilon(4 S) \rightarrow B^{0} \bar{B}^{0}\right)$. Using the ratio of the charged
TABLE II. Measured branching fractions for exclusive decays of $B$ mesons to charmonium and kaon final states. The first error is statistical and the second systematic.

\begin{tabular}{cc}
\hline \hline Channel & Branching fraction $\left(\times 10^{-4}\right)$ \\
\hline$B^{0} \rightarrow J / \psi K^{* 0}$ & $13.09 \pm 0.26 \pm 0.77$ \\
$B^{+} \rightarrow J / \psi K^{*+}$ & $14.54 \pm 0.47 \pm 0.97$ \\
$B^{+} \rightarrow J / \psi K^{+}$ & $10.61 \pm 0.15 \pm 0.48$ \\
$B^{0} \rightarrow J / \psi K^{0}$ & $8.69 \pm 0.22 \pm 0.30$ \\
$B^{0} \rightarrow \psi(2 S) K^{* 0}$ & $6.49 \pm 0.59 \pm 0.97$ \\
$B^{+} \rightarrow \psi(2 S) K^{*+}$ & $5.92 \pm 0.85 \pm 0.89$ \\
$B^{+} \rightarrow \psi(2 S) K^{+}$ & $6.17 \pm 0.32 \pm 0.44$ \\
$B^{0} \rightarrow \psi(2 S) K^{0}$ & $6.46 \pm 0.65 \pm 0.51$ \\
$B^{0} \rightarrow \chi_{c 1} K^{* 0}$ & $3.27 \pm 0.42 \pm 0.64$ \\
$B^{+} \rightarrow \chi_{c 1} K^{*+}$ & $2.94 \pm 0.95 \pm 0.98$ \\
$B^{+} \rightarrow \chi_{c 1} K^{+}$ & $5.79 \pm 0.26 \pm 0.65$ \\
$B^{0} \rightarrow \chi_{c 1} K^{0}$ & $4.53 \pm 0.41 \pm 0.51$ \\
\hline \hline
\end{tabular}

to neutral $B$ meson lifetimes $\tau_{B^{+}} / \tau_{B^{0}}=1.086 \pm 0.017$ [10], we obtain

$$
R^{+/ 0} \equiv \frac{\Gamma\left(\Upsilon(4 S) \rightarrow B^{+} B^{-}\right)}{\Gamma\left(\Upsilon(4 S) \rightarrow B^{0} \bar{B}^{0}\right)}=1.06 \pm 0.02 \pm 0.03,
$$

where the first error is statistical and the second systematic. The branching fractions have been determined using $R^{+/ 0}=1$; they are summarized in Table II. The ratios of the branching fractions for $B \rightarrow(c \bar{c}) K^{*}$ and $B \rightarrow(c \bar{c}) K$ for the three $(c \bar{c})$ states are presented in Table III. For each of the charmonium states, the average of the charged and neutral measurements is also shown.

Finally, we have measured the charge asymmetries

$$
A \equiv \frac{\mathcal{B}\left(B^{+} \rightarrow(c \bar{c}) K^{+(*)}\right)-\mathcal{B}\left(B^{-} \rightarrow(c \bar{c}) K^{-(*)}\right)}{\mathcal{B}\left(B^{+} \rightarrow(c \bar{c}) K^{+(*)}\right)+\mathcal{B}\left(B^{-} \rightarrow(c \bar{c}) K^{-(*)}\right)}
$$

using efficiencies determined separately for the two charges. The results are presented in Table IV. No statistically significant asymmetry is observed.

In summary, branching fraction measurements of exclusive $B$ decays to charmonium $\left[J / \psi, \psi(2 S)\right.$, and $\left.\chi_{c 1}\right]$ and $K$

TABLE III. Results for ratios of the branching fractions for charmonium and a $K^{*}$ versus charmonium and a $K$. The first error is statistical and the second systematic.

\begin{tabular}{cc}
\hline \hline Ratio & Result \\
\hline $\mathcal{B}\left(B^{0} \rightarrow J / \psi K^{* 0}\right) / \mathcal{B}\left(B^{0} \rightarrow J / \psi K^{0}\right)$ & $1.51 \pm 0.05 \pm 0.08$ \\
$\mathcal{B}\left(B^{+} \rightarrow J / \psi K^{*+}\right) / \mathcal{B}\left(B^{+} \rightarrow J / \psi K^{+}\right)$ & $1.37 \pm 0.05 \pm 0.08$ \\
$\mathcal{B}\left(B \rightarrow J / \psi K^{*}\right) / \mathcal{B}(B \rightarrow J / \psi K)$ & $1.44 \pm 0.04 \pm 0.06$ \\
$\mathcal{B}\left(B^{0} \rightarrow \psi(2 S) K^{* 0}\right) / \mathcal{B}\left(B^{0} \rightarrow \psi(2 S) K^{0}\right)$ & $1.00 \pm 0.14 \pm 0.09$ \\
$\mathcal{B}\left(B^{+} \rightarrow \psi(2 S) K^{*+}\right) / \mathcal{B}\left(B^{+} \rightarrow \psi(2 S) K^{+}\right)$ & $0.96 \pm 0.15 \pm 0.09$ \\
$\mathcal{B}\left(B \rightarrow \psi(2 S) K^{*}\right) / \mathcal{B}(B \rightarrow \psi(2 S) K)$ & $0.98 \pm 0.10 \pm 0.07$ \\
$\mathcal{B}\left(B^{0} \rightarrow \chi_{c 1} K^{* 0}\right) / \mathcal{B}\left(B^{0} \rightarrow \chi_{c 1} K^{0}\right)$ & $0.72 \pm 0.11 \pm 0.12$ \\
$\mathcal{B}\left(B^{+} \rightarrow \chi_{c 1} K^{*+}\right) / \mathcal{B}\left(B^{+} \rightarrow \chi_{c 1} K^{+}\right)$ & $0.51 \pm 0.17 \pm 0.16$ \\
$\mathcal{B}\left(B \rightarrow \chi_{c 1} K^{*}\right) / \mathcal{B}\left(B \rightarrow \chi_{c 1} K\right)$ & $0.65 \pm 0.09 \pm 0.10$ \\
\hline \hline
\end{tabular}


TABLE IV. Results for charge asymmetries. The first error is statistical and the second systematic.

\begin{tabular}{cr}
\hline \hline Final state & \multicolumn{1}{c}{ Asymmetry } \\
\hline$J / \psi K^{+}$ & $-0.030 \pm 0.014 \pm 0.010$ \\
$J / \psi K^{*+}$ & $0.048 \pm 0.029 \pm 0.016$ \\
$\psi(2 S) K^{+}$ & $0.052 \pm 0.059 \pm 0.020$ \\
$\psi(2 S) K^{*+}$ & $-0.077 \pm 0.207 \pm 0.051$ \\
$\chi_{c 1} K^{+}$ & $0.003 \pm 0.076 \pm 0.017$ \\
$\chi_{c 1} K^{*+}$ & $-0.471 \pm 0.378 \pm 0.268$ \\
\hline \hline
\end{tabular}

or $K^{*}$ have been presented. Our results for $J / \psi$ and $\psi(2 S)$ are in good agreement with previous measurements [10] and exhibit comparable or superior precision. Our $\chi_{c 1}$ results have much better precision than earlier measurements. The $B^{+} \rightarrow \chi_{c 1} K^{*+}$ mode was previously unmeasured. Assuming isospin invariance, we find the ratio of charged- to neutral- $B$ meson production on the $Y(4 S)$ resonance to be compatible with unity within 1.7 standard deviations. No direct $C P$ violation has been observed in the charge asymmetries.

We are grateful for the excellent luminosity and machine conditions provided by our PEP-II colleagues and for the substantial dedicated effort from the computing organizations that support BABAR. The collaborating institutions wish to thank SLAC for its support and kind hospitality. This work is supported by DOE and NSF (USA), NSERC (Canada), IHEP (China), CEA and CNRS-IN2P3 (France), BMBF and DFG (Germany), INFN (Italy), FOM (The Netherlands), NFR (Norway), MIST (Russia), and
PPARC (United Kingdom). Individuals have received support from CONACyT (Mexico), A.P. Sloan Foundation, Research Corporation, and Alexander von Humboldt Foundation.

*Also with Università della Basilicata, Potenza, Italy. †Deceased.

[1] M. Diehl and G. Hiller, J. High Energy Phys. 06 (2001) 067.

[2] BABAR Collaboration, B. Aubert et al., Phys. Rev. D 65 , 032001 (2002).

[3] CLEO Collaboration, C. Bebek et al., Phys. Rev. D 36, 1289 (1987); CDF Collaboration, F. Abe et al., Phys. Rev. D 58, 072001 (1998).

[4] ARGUS Collaboration, H. Albrecht et al., Z. Phys. C 48, 543 (1990).

[5] T. Brown, S. Pakvasa, and S. F. Tuan, Phys. Lett. 136B, 117 (1984); I. Dunietz, Phys. Lett. B 316, 561 (1993).

[6] BELLE Collaboration, K. Abe et al., Phys. Rev. D 67, 032003 (2003).

[7] BABAR Collaboration, B. Aubert et al., Nucl. Instrum. Methods Phys. Res., Sect. A 479, 1 (2002).

[8] A. Drescher et al., Nucl. Instrum. Methods Phys. Res., Sect. A 237, 464 (1985).

[9] R. Sinkus and T. Voss, Nucl. Instrum. Methods Phys. Res., Sect. A 391, 360 (1997).

[10] Particle Data Group, S. Eidelman et al., Phys. Lett. B 592, 1 (2004).

[11] BABAR Collaboration, B. Aubert et al., Phys. Rev. Lett. 87, 241801 (2001); CLEO Collaboration, C. P. Jessop, Phys. Rev. Lett. 79, 4533 (1997). 